\title{
Promoting Physical Activity in People with Functional Diversity through a Multiplayer Musical Game ${ }^{\dagger}$
}

\author{
Manuel Merino-Monge*(D), Alberto J. Molina-Cantero (D) Juan A. Castro-García (D, \\ Clara Lebrato-Vázquez (D) and Isabel M. Gómez-González (D)
}

Departamento de Tecnología Electrónica, E.T.S. Ingeniería Informática, Universidad de Sevilla, 41012 Sevilla, Spain; almolina@us.es (A.J.M.-C.); jacastro@us.es (J.A.C.-G.); clebrato@us.es (C.L.-V.); igomez@us.es (I.M.G.-G.)

* Correspondence: manmermon@dte.us.es; Tel.: +34-954-552787

† Presented at the 4rd XoveTIC Conference, A Coruña, Spain, 7-8 October 2021.

check for updates

Citation: Merino-Monge, M.; Molina-Cantero, A.J.; Castro-García, J.A.; Lebrato-Vázquez, C.; Gómez-González, I.M. Promoting Physical Activity in People with Functional Diversity through a Multiplayer Musical Game. Eng. Proc 2021, 7, 3. https://doi.org/10.3390/ engproc2021007003

Academic Editors: Joaquim de Moura, Marco A. González, Javier Pereira and Manuel G. Penedo

Published: 28 September 2021

Publisher's Note: MDPI stays neutral with regard to jurisdictional claims in published maps and institutional affiliations.

Copyright: (c) 2021 by the authors. Licensee MDPI, Basel, Switzerland. This article is an open access article distributed under the terms and conditions of the Creative Commons Attribution (CC BY) license (https:// creativecommons.org/licenses/by/ $4.0 /)$.

\begin{abstract}
Physical activity (PA) performed in group can slow down the decline in motor functions in people with disabilities. With this objective, Interactive Rehab Orchestra (IRO) was developed. IRO is an interactive multiplayer musical game that looks for reducing sedentary lifestyles by promoting PA. The individuals are responsible for playing the melody correctly. To do that, they must perform a movement when the on-screen avatar reaches a certain area. If the action is not performed, the melody will stop playing for a certain time interval. IRO is highly configurable, allowing the controller to be adapted to player skills. The customization of melodies and images is also possible according to the players' preferences, which helps to enhance player engagement. In addition, a configurable color code allows identifying when to perform an action. IRO incorporates a statistical summary to assess the evolution of the user. In this way, IRO aims at encouraging PA through music to maintain/improve muscle tone and the subjects' mobility, quantifying PA intensity, in relation to motor skills, and promoting PA so that participants can adhere to a specific program with long-term follow-up.
\end{abstract}

Keywords: physical activity; functional diversity; multiplayer musical game

\section{Introduction}

Many people with disabilities and limited motor functions, such as cerebral palsy, exhibit sedentary behavior (SB), which contributes to reducing motor functions, increasing body fat, muscle stiffness, and health issues [1]. The World Health Organization guidelines [2] recommend the practice of regular PA to reduce health risks. Three factors are important in PA: duration, frequency, and intensity. By increasing all of them, better health benefits can be achieved. Exercising in groups endows people with a higher level of engagement in the activity, allows increasing the PA, and results in an improvement in physical capabilities [3]. Additionally, the use of music to evoke emotions [4] and obtain higher levels of engagement and attention to the PA is also important [5].

This paper describes the multiplayer musical game denominated Interactive Rehab Orchestra (IRO), which aims at reducing SB lifestyles by promoting individual or group PA. The application is highly configurable and can be adapted to the user's needs, providing statistical summaries to therapists for the assessment of the players.

\section{Software}

IRO (https: / / github.com/manmermon/IRO, accessed on 22 July 2021) is a crossplatform software, developed using Java technology (version 1.8) under development but in a sufficient state so that it can be used by potential users. In this multiplayer musical game, based on midi files, the player must perform a movement when the avatar reaches a certain area. Missing actions mute the melody for a while. Therefore, the players are 
responsible for the correct sounding of the melody. IRO contains three parts: the video game itself, the controller or the user interface, and the therapist interface. As players' motor skills can be very different, IRO does not set any default controller.

\subsection{Videogame}

During the game, the user must perform a movement when the avatar reaches a certain area, such that any missing action would mute the melody for a time equal to the player's reaction time. In this way, motor capabilities, concentration, and reaction are worked on.

The game's screen (Figure 1b) shows a bar with the reached level of action (BRAL) with 3 areas (recovery level (RL) in green, action level (AL) in red, and intermediate zone in yellow) plotting the player's movement, which allows a feedback; therefore, external instructions are unnecessary. Another element is the action area. When the avatars enter this area, the player must perform the action for a limited time defined by the configurable parameter time to reach the action level (TAL). In addition, the action is split in two parts: $\mathrm{AL}$ and time at action target (TAT). If AL is not reached and maintained for TAT time, then music is muted. The local multiplayer session is implemented to increase fun and player involvement. The number of players is limited to 4 people, where each player will be assigned a specific controller, a BRAL, and a different avatar color.

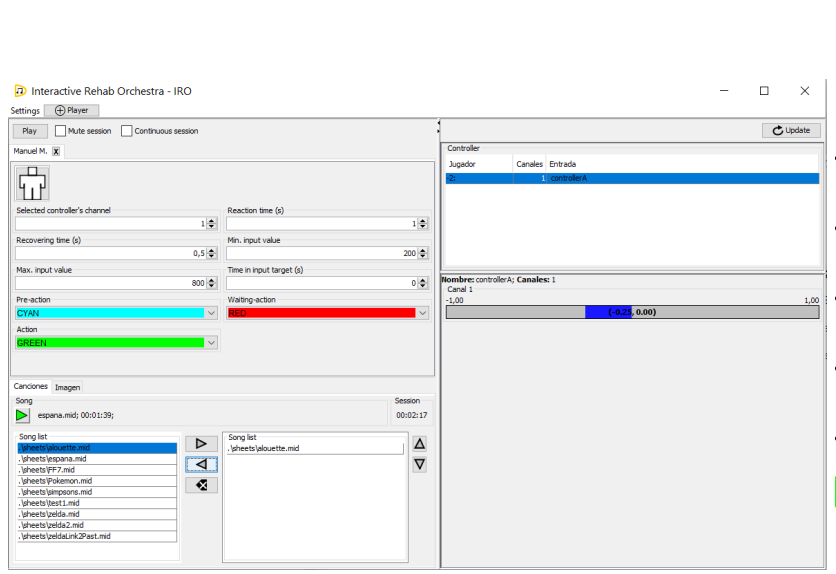

(a)

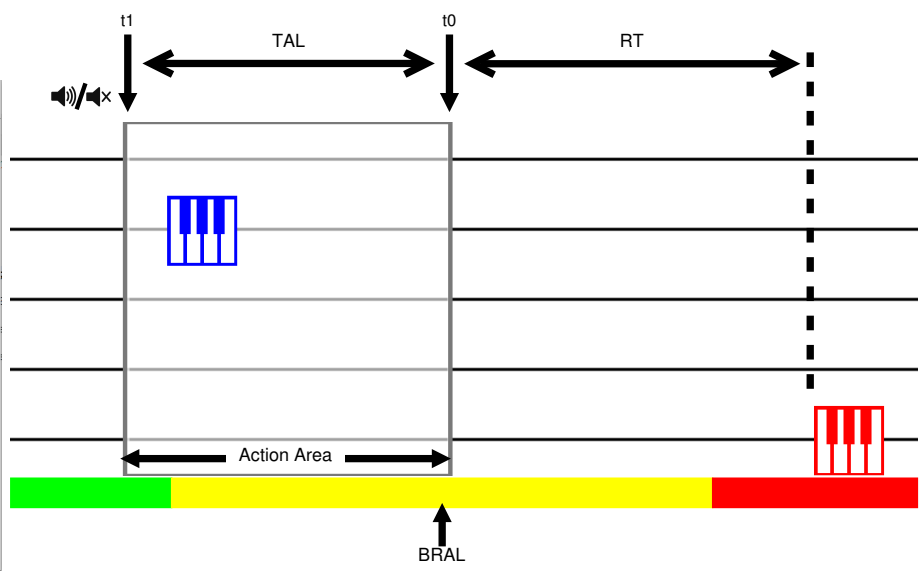

(b)

Figure 1. IRO interfaces: (a) therapist interface; (b) video-game scheme.

\subsection{Game Controller}

IRO allows using a wide range of controllers that can be adapted to players' motor skills. To achieve this, we included the Lab-Streaming Layer (LSL) library (https: //github.com/sccn/labstreaminglayer, accessed on 22 July 2021). This is a cross-platform library (Windows/Linux/MacOS and 32/64-bit computer architecture) that provides a unified interface for centralized data collection, synchronization in (near) real-time, and the ability to register multiple devices at the same time. Thus, LSL is responsible for the communication between IRO and the controller.

The therapist sets six parameters per player to interact with IRO: stream channel (SC), $\mathrm{AL}$, TAT, TAL, RL, and recovery time (RT). As any controller can send several data streams at the same time, you need to select a specific data stream. For example, a gyroscope sends the angle velocity of the $X, Y, Z$ axes associated with a movement. If you are interested in torsional motions, the stream to be used would be the one associated with the $X$ axis. Thus, the SC parameter selects exactly the control source. The AL, TAT, and TAL parameters set the range of motion to perform, which includes the time TAT to reach the preset AL level when the avatar enters the action zone, and the time TAL to remain above the level for IRO to determine that an action has been correctly performed. Finally, RL and RT 
determine the starting value to which the player must return before performing the next action (which compels subjects to perform complete movements), and the time the therapist considers enough for the player to recover, before performing the next movement (which only determines when the next note is displayed on the screen). In this way, the parameters TAL and RT determine the temporal distance between the musical notes in the game (Figure 1b).

\subsection{Therapist Interface}

The therapist interface is oriented to configure the sessions (Figure 1a). Firstly, the default music session is not set, and therapists can add new midi files. The songs must be selected so that the players' preferences can be taken into account, which will improve their engagement. The background and avatar images can be customized. Similarly, the color of the active zone can be changed to make it easier for players to identify when to perform an action. Moreover, the therapist is responsible for selecting and configuring the control interface.

In addition to these above elements, the therapist can access the statistics of each player, so that they can analyze the evolution. This is still under development. However, data logging is already implemented. Fourteen different events associated with each player are stored in a SQLite database (when an avatar is displayed/disappears on the screen, when the avatar enters/exits the action zone, when the action starts, and so on), as well as the score achieved by each player and the data of all the controller channels. With this information, the therapist can assess the evolution of the users (score, reaction time, number of errors, average time taken to reach the recovery zone in the controller, and so on).

\section{Conclusions}

IRO is a highly configurable game that is independent of any controller hardware, and that takes into consideration the preferences of the players. This application aims at encouraging PA by using music to maintain/improve muscle tone and mobility. It also allows quantifying PA levels in relation to motor skills and promoting PA so that participants can adhere to a specific program with long-term follow-up.

Author Contributions: M.M.-M. developed IRO following the advice of the other authors. All authors have contributed equally in conducting the research and writing the paper. All authors have read and agreed to the published version of the manuscript.

Funding: This research was funded by the Spanish Ministry of Science and Innovation, State Plan 2017-2020: Challenges-R\&D\&I Projects with grant code PID2019-104323RB-C32.

Conflicts of Interest: The authors declare no conflict of interest.

\section{References}

1. WHO. A Guide for Population-Based Approaches to Increasing Levels of Physical Activity: Implementation of the WHO Global Strategy on Diet, Physical Activity and Health; World Health Organization: Geneva, Switzerland, 2007; p. 20.

2. WHO. Global Priority Research Agenda for Improving Access to High-Quality Affordable Assistive Technology; World Health Organization: Genève, Switzerland, 2017; p. 24.

3. Raghavan, P.; Geller, D.; Guerrero, N.; Aluru, V.; Eimicke, J.P.; Teresi, J.A.; Ogedegbe, G.; Palumbo, A.; Turry, A. Music Upper Limb Therapy-Integrated: An enriched collaborative approach for stroke rehabilitation. Front. Hum. Neurosci. 2016, 10, 498. [CrossRef] [PubMed]

4. Yip, H.; Moore, K.S. Music Therapy for Multisensory and Body Awareness in Children and Adults with Severe to Profound Multiple Disabilities: The MuSense Manual. J. Music. Ther. 2017, 54, 479-483. [CrossRef]

5. Davies, C.; Shurdington, J.; Murray, K.; Slater, L.; Pearson, D. Music for Wellness in rehabilitation patients: Programme description and evaluation results. Public Health 2021, 194, 109-115. [CrossRef] [PubMed] 